\title{
Recenti tecniche sull'infrarosso $\left({ }^{*}\right)$
}

\author{
W. RUSPANTINI \\ Ricevuto il 19 lebbraio 1963
}

\begin{abstract}
Rrassunto. - Dopo brevi cemni storici, l'A. pone allatienzione del lettore, i fondamenti della fisica dell'infrarosso. Vengono quindi descritti le applicazioni scientifiche nel campo delle navigazione spaziale e il trasporto di strumenti specifici al limite dello spazio, i veicoli necessari per gruilarli e per racogliene i dati fisici.

SUmary. - Following a brief historical introduction, fundamentals of infrared physies are brought to the reader's attention.

Scientific applieations in space navigation field and specific instrumentation carried on board of space velicles for their guidance and for collecting phrsical data are deseribed.
\end{abstract}

In questi ultimi anni si sono andati sempre più intensificando gli studi sulle radiazioni infrarosse e veramente notevoli sono stati i progressi fatti e i risultati finora raggiunti. Ma un ben più ampio sviluppo si apre dinanzi a questa nuova branca della scienza, che lascia prevedere applicazioni sempre più importanti e di più vasta portata.

Anche nel campo spaziale, la tecnologia infrarossa offre già oggi i suoi preziosi servigi, fornendo mezzi d'indagine per le osservazioni scientifiche e la strumentazione più adatta per rendere sicura la navigazione.

L'esistenza della radiazione infrarossa è stata messa in evidenza dall'astronomo William Herschel nella primavera del 1800, si può dire quasi per caso, mentre egli stava ricercando un sistema di filtraggio della luce del sole, che gli permettesse di ottenere questa in sufficiente quantità, pur eliminando al massimo l'indesiderato effetto calorifico.

(*) Nota presentata al 20 Congresso Internazionale Teenico Scientifico dello Spazio. Roma, 19-23 Gingno 1962. 
A tal line, dopo avere seomposto la lure solare per merzo di un prisma, in una camela oscuma, egrli fece un'indagine acculata della distribuzione della temperatura sullo spettro solare visibile, usando dei normali termometri a mercurio. T'astronomo notò che la temperatura andava aumentando procedendo dal violetto al rosso, a con sua grande sorpresa, che essa continuava a crescere, anche nella zona non illuminata, dove raggiungeva un massimo.

Le ricerche furono prosegruite da suo figlio e da altri sperimentatori, che passarono dall'uso del termometro, che si ela dimostrato strumento troppo grossolano per mettere in evidenza le radiazioni, all'impiego di rivelatori molto piì sensibili. L'uso delle termocoppie, che venivano anche raggruppate in serie, consenti di misurare differenze molto piceole di temperatural .

Un ulteriore passo avanti fu fatto con l'invenzione del bolometro. Infine, con la scoperta dei fotorivelatori e con il perfezionarsi delle tecniche di fabbricazione is stato possibile oftenere cellule sempre più sensibili, che hamo permesso di indagare molto profondamente la natura e le proprietà delle radiazioni infrarosse.

Mentre i bolonetri sono rivelatori terhici, ossia mettono in evidenza l'energia totale della radiazione che cade su di essi, i fotorivelatori si basano sulla liberazione di un elettrone, per ogni singolo quanto di radiazione che da essi viene assorbito.

Lat differenza essenziale fra i fotorivelatori a i detector termici risiede nella circostanza, che mentre i primi sono sensibili solamente al numero effettivo di quanti assorbiti, i secondi rispondono all'energia totale ricevuta.

Tenendo presente che l'energia di un quanto e data dal prodotto della sua frequenza di vibrazione per la costante $h$ di Planck, si nota che i fotorivelatori mettono in evidenza i quanti, che hamno un'energia più grande di un resto valore limite ed ignorano completamente tutti gli altri quanti, che hanno un valore più basso. A tale valore limite di energia di attivazione, (anatteristico di ogni tipo di detector, rorrisponde una frequenza e quindi una lunghezza d'onda limite ben determinata.

Per i detertor fotoemissivi, rioè quelli in eni arviene l'espulsione degli elettroni, la lunghezza d'onda linite è di circa $1,3 \mu$.

Rivelatori di tale tipo, aventi un fotocatodo costituito da Cs-O-Ag, hamno trovato impiego per mettere in evidenza radiazioni situate nell'infrarosso vieino. Gli elettroni emessi vengono sfruttati per la visione notturna passiva. Essi vengono messi in evidenza sulla lastma fuorescente e forniscono rosi l'immagine della zona osservata. 
Tali apparecehi sono sensibili per quanto si c. detto, solo a frequenze elevate e quindi in una stretta gamma dellinfrarosso molto vicino. Essi possono rilevare oggetti notevolmente (aldi, ovvero (he siano "illuminati) da ma sorgente di radiazioni infrarosse.

Fortumatamente, per superare tale difficolta, viene in soceorso un altro tipo di fenomeno fotoelettrico, che ha avuto un effetto straordinario nello sviluppo dei rivelatori per infrarossi e che si manifesta in quella particolare categoria di corpi, che per la loro proprietà caratteristica, vengono detti semiconduttori. Elettroni liberi possono essere prodotti nell'interno di tali sostanze, con l'assorbimento di appropriati quanti di radiazione. Inoltre, non essendo più necessario disporre di ma quantità notevole di energia, per provocare l'espulsione dell'elettrone, ma solo una frazione di essa, si è riusciti a mettere in evidenza, con il continuo perfezionarsi di tali rivelatori, radiazioni aventi lunghezze d'onda sempre più lunghe.

La printa sostanza ad essere impiegata è stata il solfuro di tallio. Esso fu introdot to nel 1920 e messo in uso facendolo depositare in sottile strato, su di una lastrina di vetro.

Vemnero poi il solfuro di piombo, il telluriuro di piombo, il seleniuro di piombo, l'antimoniuro d'indio ed altri composti, che hamno permesso, impiegando particolari tecniche di fabbricazione, di ottenere materiali molto sensibili.

Inoltre raffreddando le cellule alle temperature di liquefazione dell'azoto, dell'idrogeno o di altri gas, la sensibilità è stata noterolmente aumentata, fino a consentire la rivelazione di lunghezze d'onda di 9u.

Un altro grande passo avanti è stato fatto con il successivo perfezionarsi della teenica dei semiconduttori.

I cristalli di germanio, attivati con impurità di oro, rame, zinco ed antimonio hanno consentito di arrivare alla rivelazione di radiazioni, aventi lunghezze d'onda sempre più lunghe. Impurità di zinco aggiunte al germanio, permettono infatti di rivelare lunghezze d'onda fino a $40 \mu$, mentre impurità di antimonio, portano il limite a $130 \varkappa$.

All'inizio della seconda Guerra Mondiale le radiazioni infrarosse, impiegate nella teconica spettroscopiea, trovavano applicazione nell'industria dei petroli e specialmente in quella chinnica, per l'analisi delle varie sostanze.

Inoltre, specie in Germania, era stata riconosciuta l'importanza delle possibili applieazioni di tali radiazioni ed ma serie di studi e di ricerche venivano effettuati nei campi fondamentali riguardanti l'emissione, l'attenuazione atmosferica e la radiazione emessa dal background. 
Con lo scoppio dell'ultima guerra le ricerche venivano febbrimente intensificate, con notevoli risultati. Ise mdiazioui infurasse venivano applicate in sistemi di commicazione e di segnalazione, di guida dei missili e per la scoperta ed inseguimento di bersagli aerei e navali.

Venivano prodotti specialmente rivelatori fotoconduttivi al solfuro di piombo, con i metodi di precipitazione chimica o di evaporazione. Venivano inoltre sviluppate le tecniche per aumentare la sensibilità di tali cellule e venivano costruiti tubi convertitori d'immagine, che erano in grado di rendere perfettamente visibile un bersaglio riflettente radiazioni infrarosse.

Grande importanza acquistavano anche le applicazioni della fotogratia a raggi infrarossi, lo sviluppo delle emulsioni adatte a tale scopo e del materiale oftico.

Analogo lavoro di ricerea veniva effettuato dagli Nlleati, che a loro volta mettevano in produzione apparecchiature per la visione notturma e per la guida di automezzi nell'oseurità.

Dopo la guerra venivano sempre più perfezionati gli spettrometri, gli strumenti di ricerca, i sistemi di guida per i missili e veniva effettuato un intenso studio sistematico in tutti i più svariati campi, che hamno attinenza con la tecnica degli infrarossi.

Il lavoro di ricerca in questi ultimi anni è divenuto sempre più intenso. Schiere di ingegneri, di fisici, di scienziati, sono alla ricerca continua di nuovi metodi e perfezionamenti, nei vari istituti scientifici e nelle società produttrici americane; schiere ugualmente operose lavorano in Inghilterra, in Francia, nel Canadà, in Svezia, nei Paesi Bassi, nel Belgio, ece.

In Italia non è stato dato finora un impulso adeguato in questo settore ed è auspiabile che anche nel nostro Paese vengano al più presto promossi studi e ricerche. Occorre riguadagnare il tempo perduto e mettersi al passo con le alt re nazioni, per non venire irrimediabilmente distaccati. Bisogna incoraggiare l'industria, favorire il perfezionamento dei tecnici e formare una mentalità dell'infrarosso.

Per lo sviluppo di un tale processo sono necessari naturalmente mezzi adeguati, ma la spesa, data l'importanza dell'argomento, verrà un domani fortemente remunerata.

$$
* * *
$$

La radiazione infrarossa è generata sia dalla transizione di elettroni tra due livelli energetici, sia da cambiamenti degli stati vibratori e di rotazione degli atomi e delle molecole che si trovano in ogni sostanza. Allorché gli elettroni passano da un livello di energia ad altri più bassi, 
si ha un'emissione di radiazioni appartenenti allo spettro visibile ed all'infrarosso molto vicino. Quando ha luogo un cambiamento dello stato vibratorio, la radiazione è situata nella regione infrarossa fino a circa $30 \mu$, mentre se ha luogo un cambiamento della sola rotazione, lo spettro è situato nell'infrarosso lontano.

La banda di frequenza della radiazione infrarossa è compresa approssimativamente fra un milione a 400 milioni di megacicli, ossia fra la regrione delle microonde e quella della luce visibile.

Lo spettro della radiazione infrarossa, in termini di lunghezza d'onda ì compreso fra $0,72 \mu$ e $1000 \mu$, ossia fino a un millimetro.

Di conseguenza la radiazione infrarossa presenta alcune proprietà caratteristiche della luce, ed altre delle radioonde. Infatti può venire otticamente focalizzata mediante lenti o riflessa per mezzo di specehi, come pure trasmessa similmente alle onde radio o radar, attraverso materiali opachi alla luce visibile.

In relazione ai particolari sistemi di rivelazione usati, lo spettro è stato diviso in infrarosso vicino, medio e lontano.

I sistemi ad infrarosso possono inoltre largamente dividersi in due grandi categorie: attivi e passivi. Nei sistemi attivi è necessario l'impiego di una sorgente ausiliaria di infrarossi, per cui l'oggetto da individuare rinvia per riflessione la radiazione sul rivelatore; nei sistemi passivi la ladiazione invece è quella naturalmente emessa dall'oggetto stesso da individuare.

Mentre i sistemi attivi vamno diminuendo d'importanza pratica, in quanto la sorgente ausiliaria di raggi infrarossi, in caso di impiego bellico, è anche facilmente individuabile da parte del nemico, quelli passivi incontrano sempre più crescente favore, non essendo soggetti a tale limitazione.

Nei confronti dei sistemi radar, gli infrarossi hanno inoltere notevoli vaniaggi. Infatti, dato che la frequenza degli infrarossi è di gran lunga più alta delle onde elettromagnetiche impiegate nei radar, si ha la possibilità di oftenere un notevole dettaglio degli oggetti rilevati, con il vantaggio che non è necessario disporre della sorgente ausiliaria trasmittente.

Per illustrare la pronunciata capacità risolutiva del sistema an infrarossi, nei confronti di quello radar, basterà ricordare come classico esempio, che mentre quest'ultimo è in grado di distinguere separatamente due aerei a 8 chilometri di distanza e che volano a 400 metri uno dall'altro, il sistema ad infrarossi è capace di discernere separatamente addirittura i loro motori.

In paragone al radar, i sistemi ad infrarossi inoltre sono relativamente semplici, di ingombro limitato, leggeri e di basso costo. Si è visto infatti, 
che in missili in grado di assolvere missioni simili, il sistema ad infrarossi costa dieci volte meno di quello radar.

Ini converso è fastidioso per gli infrarossi l'assorbimento da parte dell'atmosfera (nuvole, pioggia, nebbia), che alle basse quote può anche essere di una certa importanza.

Alemi costituenti atmosferici contengono cariche di legame, con frequenze naturali di vibrazione e rotazione, che dipendono dalla particolare costituzione della molecola. Quando queste frequenze caratteristiche si accordano con quelle della padiazione incidente, si manifesta l'assorbimento per risonanza. Ne risulta un'ampiezza di vibrazione più grande e l'energia assorbita viene reirradiata in tutte le direzioni. In effetti perciò, in una direzione particolare, la radiazione incidente è attenuata o assorbita.

Ma per le applieazioni relative alla navigazione spaziale, il problema della trasmissione atmosferica praticamente non esiste, in quanto alle quote elevate il cielo è completamente trasparerite.

Amalogamente ai telescopi ottici, gli apparecehi ad infrarossi sono capaci di "vedere s a grande distanza e misur"are le direzioni e gli angoli ron struordinaria precisione.

Siccome nel eampo dell'infrarosso tutti gli oggetti sono "luminosi " per sé stessi, non occorre che siano illuminati artificialmente. Cosicché la luna, la terra e gli altri pianeti, anche se si trovano in ombra diventano visibili se all'ocehio mano viene sostituito l'adatto strumento.

L'astronauta che naviga nello spazio deve per prima cosa conoscere la sua esatta posizione, il suo orientamento e la propria velocità, onde possa essere eserritata sugli strumenti di governo la dovuta azione, per il raggiungimento dell'obbiettivo prefisso. Se intende manovrare il suo veicolo spaziale mediante razzi o getti, egli deve conoscere esattomente il suo asset to rispetto alla direzione del suo moto.

Tel caso di veicoli spaziali senza persone a bordo, quanto sopra assume un'importanza decisiva, dato che tutte le azioni e le decisioni, debbono essere operate in maniera completamente automatica.

La terra, circondata dalla sua fascia atmosferica, è vista da un satellite nello spazio, come un corpo notevolmente caldo, la cui temperatural assoluta è in netto contrasto con quella dello spazio profondo.

Il limite dell'orizzonte apparente è situato a una decina di chilometri al disopra della superficie terrestre, con una incertezza che d̀ dello stesso ordine di quella dovuta alle protuberanze terrestri a alla differenza tra i raggi ai poli a all'equatore. 
Da un satellite che si trova in un orbita a $750 \mathrm{~km}$ di altezza, il bordo di tale fascia viene a sottendere $\mathrm{m}$ angolo di circa $0,3^{\circ}$. Dentro tale angolo veramente piccolo, l'orlo dell'orizzonte appare ad un sistema di rivelazione ad infrarossi, molto ripido, con un salto di temperatura che ì di circa $250^{\circ} \mathrm{K}$.

Approfittando di tale circostanza nei recenti lanci spaziali, la capsula Mereury è stata dotata per il suo orientamento, di un perfezionato strumento ad infrarossi, la cui funzione ì stata di importanza decisiva all'atto del rientro nell'atmosfera.

Per rilevare con sicurezza la discontinuità dell'orizzonte della terra rispetto allo spazio circostante, lo strumento ì stato reso insensibile alla luce solare riflessa, impiegando un filtro di germanio puro, che elimina tutte le lunghezze d'onda più corte di $1,8 \mu$, ossia circa il $95 \%$, dell'energia dovuta all'effetto solare.

Per stabilire la verticale locale, necessaria all'orientamento del veicolo spaziale, il sistema sensorio effettua contemporaneamente due scansioni coniche, ortogonali fra loro, con un ristret to campo di vista di $2^{\circ} \times 8^{\circ}$ ed un angolo di apertura di $110^{\circ}$.

Lal scansione viene effettuata a 30 giri al secondo e mette in marcata evidenza ogni passaggio attraverso l'orizzonte, data la difierenza di temperatura esistente tra la terra e lo spazio.

Le uscite dei rivelatori producono segnali ad onda rettangolare, ('he' vengono confrontati con altri segmali genemati internamente allo strumento. La differenza di fase fra i segnali in arrivo e quelli campioni, permettono di determinare con esattezza l'angolo di becoheggio e di rollio.

La cellula impiegata è costituita da un rivelatore termico a base di ossidi metallici. Non è stato usato un elemento fotoconduttivo, per esempio uno dei più recenti tipi di detector a base di germanio attivato, dato che questo avrebbe richiesto mu raffredkamento molto spinto e quindi, oltre la inevitabile complicazione, avrebbe rappresentato anche un notevole peso supplementare.

Un altro apparecchio basato sulla tecnica degli infrarossi, verrà montato sul veicolo spaziale Mariner, destinato all'esplonazione dei piancti Sirte e Venere. Tale strumento consentirà in un prino tempo di localizzare ad esempio uno di questi due pianeti da $150.000 \mathrm{~km}$ di distanza, per mezzo di un sistema di scansione a ruggi infrarossi e fornirà sucecessivamente l'orientazione fino a circa $8000 \mathrm{~km}$ di distanza.

Il rilevamento della posizione del veicolo spaziale in relazione al pianeta, al Sole ed alla Terra, è fondamentale per la esattezza della raccolta e trasmissione dei dati. Infatti il sistema deve essere in grado di 
dirigere verso il Sole le batterie solari, capaci di produrre una potenza di 300 Watt, e di puntare le antenne altamente direzionali sulla Terra.

Il programma comprenderà sette missioni interplanetarie, tutte intorno a Venere e a Marte. Il primo lancio di un prototipo di veicolo spaziale è previsto per il 1963 e tale prova preluderà una missione attorno a Marte per il 1964 .

Elevatissima dovà essere la sensibilità degli strumenti sensori del veicolo spaziale, in quanto le radiazioni giungeranno ad esso in quantità molto morlesta.

A causa delle basse temperature delle superfici emittenti, l'energia disponibile per la navigazione sarà quella corrispondente ad onde molto lunghe. Venere emette infatti dalla sommità della sua densa atmosfera che si trova a $225^{\circ} \mathrm{K}$, lunghezze d'onda aventi il massimo di emissione a $11, \tilde{\delta} \mu$

Varte, la cui atmosfera è piuttosto mefatta, emette invece dalla sua superficie nella zona equatoriale, che si trova a $280^{\circ} \mathrm{K}$, lunghezze d'onda aventi il massimo di emissione a $10 \mu$ e dalle zone polari, che si trovano a $205^{\circ} \mathrm{K}$, lunghezze d'onda aventi il massimo di emissione a $11 \mu$.

11 prin:o compito dello strumento sensorio è quello d'individuare il pianeta. Per far ciò, due prismi controrotanti a velocità diversa esplorano mediante un campo di vista di $1 / \underline{2}^{0} \times 1 / \underline{y}^{0}$, un luogo geometrico a forma di quadrifoglio, che è contenuto in un cono avente un'apertura totale di 700. Il quadrifoglio inoltre viene fatto ruotare lentamente, finché il borsaglio viene individuato. In tale istante il motore di acquisizione si ferma e le radiazioni infrarosse raccolte, vengono trasformate in tensioni elettriche, per mezzo del detector. Misurando la durata delle tensioni ed i tempi ad essa relativi, il sistema dà luogo ad un segnale di errore, in corrispondenza di eiascruna foglia.

Allorché mediante opportuna orientazione, questi errori vengono resi tra loro uguali, il pianeta viene a trovarsi in corrispondenza del centro del quadrifoglio.

Un altro importante strumento basato sulla tecnica degli infrarossi. è stato montato sui satelliti Tiros, che sono stati lanciati negoli USA dalla NASA per osservazioni meteorologiche.

Si tratta di un particolare radiometro a 5 eanali, per la misura di altrettante bande spettrali, che impiega come riferimento, anziché un corpo nero campione, la radiazione proveniente dallo spazio e che è prossima allo zero assoluto. Nello strumento la faccia di un prisma viene diretta verso lo spazio, dove la temperatura è praticamente costante e un'altra faceia in direzione della terra. Uno specehio ruotante, che ha metà 
della sua superficie ricoperta di sostanza assorbente gli infrarossi, riceve su una metà la radiazione da misurare. La rotazione di questo specechio rende alternato l'invio della ladiazione alla cellula bolometrica, di guisa che il risultante segnale in corrente alternata, fornisce la misura della differenza tra la radiazione di riferimento e quella osservata.

I 5 canali operano tutti in questa maniera. Le loro uscite vengono poi amplificate, rettificate a raccolte su un nastro magnetico, per venire ritrasmesse a comando.

Il primo canale raceoglie l'emissione di acqua-vapore intorno ai $6 \mu$; il secondo la luce visibile $(0,55 \div 0,75 \mu)$; il terzo la radiazione solare $(0,2 \div$ $5,5 \mu)$; il quarto l'emissione totale della terra $(7,5 \div-30 \mu)$; il quinto, quale finestra atmosferica, misura la temperatura della terra o della coltre di nuvole che la ricopore.

Un altro ladiometro a due canali is stato inoltre montato sul satellite e permette di determinare il rapporto fra l'energia infradossa riflessa dalla ferral e quella della radiazione solare totale ricevuta.

$$
* * *
$$

Radiometri particolarmente sensibili permettono di determinare e misurare dalla terra le radiazioni provenienti dai lontani pianeti e di risalire alla loro temperatura.

E stato cosi possibile determinare la temperatura della Luma, che quanclo è completamente illuminata dal Sole, raggiunge i 120 of e secende a --150 or quando si trova in ombra.

Similmente sono state determinate le temperature degli altri corpi celesti del sistema solare, che vanno da 400 or nel caso di Mereurio a -150 oC per Saturno.

Il rilievo della temperatura di corpi che si trovano a distanze cosi elevate è possibile usando i mezzi della radiometria dell'infrarosso, in quanto la radiazione totale emessa da un corpo è una funzione ben nota della sua temperatura assoluta.

I satelliti messi in orbita, possono ugualmente renire localizzati da ferra ed inseguiti da perfezionati strumenti, che ne mismano la radiazione emessia.

Radiometri costruiti secondo le tecniche piò recenti sono in grado di eflettuare rilevazioni di bersagli piccoli e lontani, in rapido movimento, su una varietà di sfondi notturni e diumi. Sistemi di scansione a reticolo, respingono i segnali uniformi del fondo (filtraggio spaziale), permertendo che misure di contrasto possano venire effettuate durante il giorno, 
allorehé la radiazione del bersaglio è mascherata dalla radiazione del cielo.

Apparecebi di tracking automatici all'infra rosso agganciano e seguono missili e velivoli veloci e forniscono gli errori in azimuth ed in elevazione ad un servo-piedistallo, che punta la sua linea di mira e quella di ogni strumento collegato, accularatamente sul bersaglio in movimento.

Telle installazioni a terra tali sistemi vengono usati anche come sicurezza di poligono per seguire i missili durante le fasi iniziali di volo, in rui il radar è ostacolato dal chutter del terreno.

Per lo studio del rientro dei missili nell'atmosfera terrestre, gli strumenti di cui si è fatto cenno, trovano la loro piò naturale applicazione.

Neggli USA, allo scopo di investigare le caratteristiche fisiche del rientro dei missili balistici, una nave ò stata trasformata in osservatorio galleggiante ed attrezzata con una grande varietà di strumentazione, comprendente apparecohi radar, inseguitori ad infrarosso e strumenti ottici. 